\title{
Combined Effect of Aerobic and Resistance Interval Training on Ejection Fraction in Myocardial Infarction
}

\author{
Hania Farheen1, Zara Khalid2, Muhammad Iqbal Tariq³, Tahira Sadiq4, Imran Amjad³ and Tahir Ramzan³
}

\begin{abstract}
This study was conducted to determine whether combination of aerobic and resistance interval training had superior effects than aerobic interval training alone on ejection fraction, cholesterol, and triglycerides in myocardial infarction patients, at Pakistan Railway General Hospital Rawalpindi from July to December 2016. Patients were randomly allocated in interventional $(n=13)$ and control group $(n=13)$ using toss and trial method. Aerobic interval training at $65 \%-85 \%$ of target heart rate was performed in three intervals in both groups; whereas, resistance training at $30-50 \%$ of one repetition maximum was added in experimental group. The outcomes were measured before and after six weeks. Ejection fraction was significantly $(p=0.029)$ improved in interventional group 55 (10) as compared to control group 50 (5). Cholesterol levels also showed significant decrease $(p=0.021)$ in control group. The study concluded that combined training has superior effect in improving ejection fraction; whereas, aerobic interval training alone is more effective than combine training in improving cholesterol.
\end{abstract}

Key Words: Cholesterol, Heart rate, Pakistan, Resistance training, Stroke volume, Triglycerides.

Annually about 17 million deaths are caused by cardiovascular diseases worldwide. 1 Globally myocardial infarction (MI) is the established leading cause of morbidity and mortality. ${ }^{1}$ Ejection fraction (EF), which is clinical predictor of long term mortality and marker of myocardial contractility, is significantly reduced after MI.2

Cardiac rehabilitation is well established and costeffective treatment. ${ }^{3}$ Patients receiving cardiac rehabilitation along with medical treatment have a survival rate of $95 \%$ at 3 years, as compared to $64 \%$ of those who are on medical treatment only. ${ }^{4}$ Resistance training combined with aerobic training is now considered integral part in rehabilitation of patients with MI.1 Recently, interval training is introduced in coronary artery disease (CAD) patients. ${ }^{1}$ Recent literature supports that aerobic interval training (AIT) alone or in combination with resistance has no safety hazards and is more effective than continuous moderate intensity training in improving EF, stroke volume, and risk factors. ${ }^{3}$

1 Department of Rehabilitation Science, Shifa Tameer Millat University, Shifa College of Rahabilitation Sciences,

Islamabad, Pakistan

2 Department of Rehabilitation Science, Foundation University, Foundation Institute of Rahabilitation Sciences, Islamabad, Pakistan

3 Department of Rehabilitation Science, Riphah International University, Riphah College of Rahabilitation Sciences, Islamabad, Pakistan

4 Department of Cardiology, Rawalpindi Institute of Cardiology, Rawalpindi, Pakistan

Correspondence: Dr. Imran Amjad, Department of Rehabilitation Science, Riphah International University, Riphah College of

Rahabilitation Sciences, Islamabad, Pakistan

E-mail: mianimran.pt@gmail.com

Received: March 06, 2018; Accepted: November 16, 2018
Many studies conclude that combination of aerobic and resistance interval training has positive effect on EF. To the best of authors' knowledge, there has been no study on head to head comparison of these two cardiac rehabilitation techniques. Moreover, outcome of EF was mostly seen in heart failure patients. Therefore, the purpose of the study was to determine whether combination of resistance training with AIT has superior effects on these outcomes for its applicability in outpatient cardiac rehabilitation programmes.

This study was conducted on 26 stable post-MI patients at Pakistan Railway General Hospital Rawalpindi, Pakistan from July to December 2016, after obtaining necessary administrative and ethical approvals. Nonprobability convenient sampling was used and grouping was done using toss and trial method. Informed consent was taken from each participant prior to participation. Stable post MI patients with first episode of MI, age >35 years and who remained asymptomatic for first three minutes of treadmill walk test were included. Patients with LVEF below $35 \%$, cardiac surgeries, arrhythmias or lung diseases were excluded. EF and lipid profile were primary and secondary outcomes, respectively. Echocardiography, cholesterol and triglycerides tests were done pre- and post-treatment. Pre- and post-exercise vital signs along with dyspnoea and fatigue levels were measured using modified Borg scale and numerical fatigue rating scale, respectively. In all patients, $65-85 \%$ of target heart rate (THR) was calculated using Karvonen's formula. 1

Patients in the control group performed three sets of aerobic exercises at intensity of $65-85 \%$ of THR in intervals. Patients performed six minutes of cycling in the first two interval sets and six minutes of treadmill in the third set. Resistance training at an intensity of $30-50 \%$ 
of 1 repetition maximum ( 1 set of 10-12 reps) was added in experimental group. Patients in the first interval performed three minutes of cycling followed by 1.5 to $2.5 \mathrm{lbs}$ hand grippers and 1-3 kgs of biceps resistive exercises. In second interval patients performed three minutes of cycling and 1-3 kgs of quadriceps resistive exercises. In the last interval, they performed three minutes of treadmill walk at the starting speed of $3 \mathrm{~km} / \mathrm{hr}$ followed by ankle pumps. Each interval was followed by three minutes of complete rest. In both groups, patients performed six weeks of training for three days per week on alternating days. Intensity was gradually increased weekly, according to each patient's THR. Likewise, weights and frequencies were increased with progressive weeks. This protocol was taken from previous systemic review. ${ }^{1}$ Slight modification of the protocol was done with the help of supervisor after presenting pilot study of four patients in front of senior research committee of university, which included cardiologist, cardiopulmonary rehabilitation specialist and $\mathrm{PhD}$ physiotherapist.

CONSORT Flow Diagram was given to share further details of the study (Figure 1). Shapiro-Wilk test of normality was applied on baseline on EF and lipid profile, $p$-value $<0.05$, representing that data is not normally distributed, so test of choice was nonparametric. Median (IQ) was calculated for quantitative variables (EF and lipid profile) except of age, which was calculated by mean $\pm S D$. Independent sample T-test was applied on age to analyse difference between interventional and control group. Qualitative variables, such as gender, occupation, and comorbidities were expressed as frequencies and percentages. To analyse the difference in outcome measure due to intervention, Mann-Witney U-test was used. Data was analysed by using SPSS version 21.0.

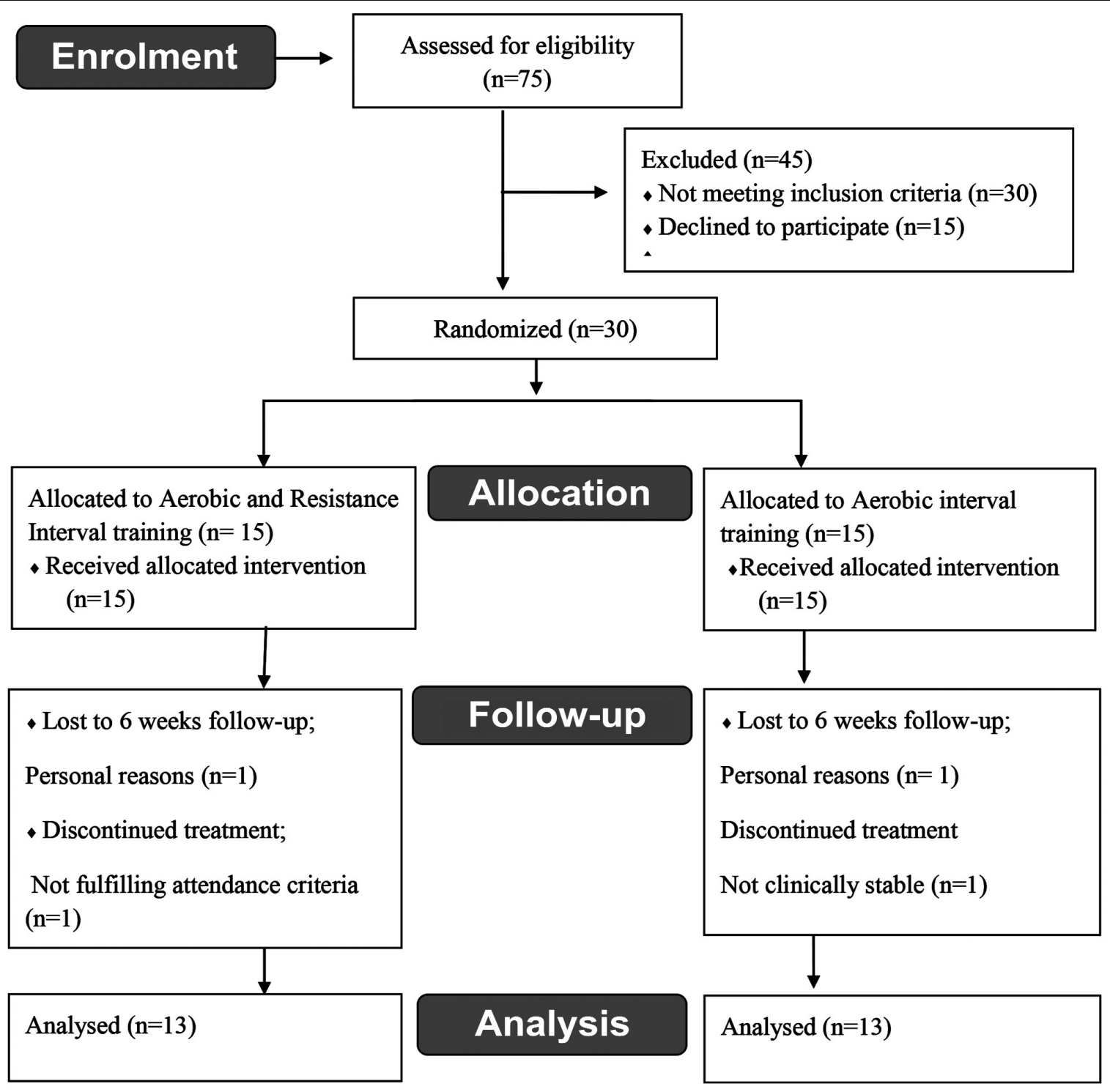

Figure 1: Consort flow diagram. 
Table I: Comparison of pre- and post-exercise cholesterol and triglycerides between the groups.

\begin{tabular}{|c|c|c|c|c|c|c|}
\hline \multirow[t]{2}{*}{ Variable } & \multicolumn{2}{|c|}{ Interventional group } & \multicolumn{2}{|c|}{ Control group } & \multirow[t]{2}{*}{ Z-value } & \multirow{2}{*}{$\begin{array}{c}\mathrm{p} \text { - value } \\
\text { Mann-Whitney U-test }\end{array}$} \\
\hline & Median (IQ) & Mean rank & Median (IQ) & Mean Rank & & \\
\hline \multicolumn{7}{|l|}{ Cholesterol (mg/dl) } \\
\hline Pre-treatment & $153(47)$ & 9.42 & $190(41)$ & 17.58 & -2.720 & $0.007^{*}$ \\
\hline Post-treatment & $127(57)$ & 10.05 & $160(31)$ & 16.96 & -2.313 & $0.021^{*}$ \\
\hline \multicolumn{7}{|c|}{ Triglycerides (mg/dl) } \\
\hline Pre-treatment & $169(151)$ & 12 & $202(123)$ & 15 & -1.001 & 0.317 \\
\hline Post-treatment & $116(122)$ & 11.77 & $174(119.5)$ & 15.23 & -1.155 & 0.248 \\
\hline
\end{tabular}

${ }^{*} p$-value $<0.05$

A total of 26 patients were enrolled that included $7(53.8 \%)$ and $9(69.2 \%)$ males and $6(46.2 \%)$ and $4(30.8 \%)$ females in experimental and control group, respectively. There were no significant difference $(p=0.716)$ in age: the mean of the interventional (57.23 \pm 9.76 years) and control groups ( $55.77 \pm 10.46$ years). In both groups, majority of patients were having a sedentary lifestyle. Hypertension was the most common comorbidity in the control group, 5 (38.5\%); whereas, hypertension and diabetes were equally present $(4,30.8 \%)$ in interventional group.

Mann-Whitney U-test was applied on pre- and posttraining EF of Ml patients for comparison between the interventional and control groups. EF before treatment of interventional 45 (15) and control group 45 (10). After six weeks of treatment, EF was significantly $(p=0.029)$ improved in interventional group $55(10)$ as compared to control group 50 (5). Cholesterol levels also showed significant $(p=0.021)$ decrease in the control group (Table I).

In the present study, greater improvement of EF compared to control group was in accordance with a recent RCT by Christina et al. that supported the concept that factors responsible for increasing EF and stroke volume improved by combined aerobic interval and resistance training. ${ }^{3}$ One of the possible explanations of greater increase in our study with resistance training might be due to the reason that combined training resulted in greater improvement of endothelial and autonomic functions due to greater generation of large shear forces. ${ }^{3}$ This might resulted in greater decrease in after load, which in turn increased EF and stroke volume to greater amount as compared to aerobic training alone. ${ }^{3}$

This study found that cholesterol levels improved in both groups after training with greater improvement in control group; whereas, post training triglycerides showed no improvement in both groups. Katharine et al. reported similar results that high intensity aerobic interval and resistance training did not improve lipid profile. ${ }^{5}$ The findings in the present study are also supported by a systemic review. ${ }^{6}$ The study concluded that AIT is more effective in improving high density lipoprotein (HDL), resistance training in lowering low density lipoprotein (LDL); whereas, combined training can improve both, while the effect on total cholesterol and triglycerides are minimum in all training programmes. 6

This study, on a small sample of 26 Pakistani patients, concludes that patients with MI should be provided combination of aerobic and resistance interval training in order to have an improved EF. Further, it concluded that combined training has no superior effects in improving cholesterol and triglycerides; whereas, aerobic interval training alone is effective in improving cholesterol.

Age range was the limitation of the study because of narrow inclusion criteria. It is recommended that studies with larger sample size and extended weeks of training should be conducted to see whether they provide greater benefits in improving these outcomes.

\section{REFERENCES}

1. Cornish AK, Broadbent S, Cheema BS. Interval training for patients with coronary artery disease: A systematic review. Eur J Appl Physiol 2011; 111:579-89.

2. Meta-analysis Global Group in Chronic Heart Failure (MAGGIC). The survival of patients with heart failure with preserved or reduced left ventricular ejection fraction: an individual patient data meta-analysis. Eur Heart $J$ 2012; 33: 1750-7.

3. Chrysohoou C, Angelis A, Tsitsinakis G, Spetsioti S, Nasis I, Tsiachris D, et al. Cardiovascular effects of high-intensity interval aerobic training combined with strength exercise in patients with chronic heart failure. A randomized phase III clinical trial. Int J Cardiol 2015; 179:269-74.

4. Williams MA, Ades PA, Hamm LF, Keteyian SJ, La Fontaine TP, Roitman JL, et al. Clinical evidence for a health benefit from cardiac rehabilitation: an update. Am Heart J 2006; 152:835-41.

5. Currie KD, Bailey KJ, Jung ME, McKelvie RS, MacDonald MJ. Effects of resistance training combined with moderate-intensity endurance or low-volume high-intensity interval exercise on cardiovascular risk factors in patients with coronary artery disease. J Sci Med Sport 2015; 18:637-42.

6. Tambalis K, Panagiotakos DB, Kavouras SA, Sidossis LS. Responses of blood lipids to aerobic, resistance, and combined aerobic with resistance exercise training: A systematic review of current evidence. Angiology 2009; 60:614-32. 